\title{
The Current Status and New Research Progress of Keratoconus Treatment
}

\author{
Ren Fengyue ${ }^{1}$, He Shuxi ${ }^{2}$ \\ ${ }^{1}$ Department of Medical College, Hunan Provincial People's Hospital, The First Affiliated Hospital of Hunan Normal University, Changsha, \\ China \\ ${ }^{2}$ Center for Ophthalmic Optics, Hunan Provincial People's Hospital, Changsha, China
}

Email address:

861801682@qq.com (Ren Fengyue), shuxi9918@163.com(He Shuxi)

\section{To cite this article:}

Ren Fengyue, He Shuxi. The Current Status and New Research Progress of Keratoconus Treatment. International Journal of Ophthalmology \& Visual Science. Vol. 4, No. 1, 2019, pp. 14-18. doi: 10.11648/j.ijovs.20190401.13

Received: January 28, 2019; Accepted: March 13, 2019; Published: April 13, 2019

\begin{abstract}
Keratoconus is a common non-inflammatory, bilateral progressive corneal dilatation disease, often leading to progressive thinning of the corneal stromal layer, the central region of the cornea is conical, clinical manifestations of high myopia and irregular astigmatism, The normal life of the patient is greatly inconvenient. Therefore, timely and effective treatment is extremely important to improve the visual quality of patients and their physical and mental health. In the early stage of the patient, vision correction can be performed by wearing a frame mirror or a contact lens; as the lesion progresses, the former is insufficient for correction, and corneal stroma implantation, corneal collagen cross-linking, and keratoplasty are feasible. Numerous studies have confirmed that this treatment can effectively improve and maintain the visual quality of patients. In recent years, new treatment methods such as matrix regeneration, matrix lens transplantation and scleral mirror have gradually become research hotspots at home and abroad. This article reviews the current major treatments for keratoconus and its new developments.
\end{abstract}

Keywords: Keratoconus, Treatment Method, Research Progress

\section{Introduction}

The keratoconus is a corneal dilatation disease characterized by progressive thinning of the corneal stroma and conical protrusion in front of the cornea. The main clinical manifestations are progressive deepening of myopia and irregular astigmatism, with varying degrees of corrected visual acuity. The keratoconus usually begins in adolescence, is common in 15-25 years old, and gradually stabilizes when it is more than 40 years old. Domestic and foreign surveys show that the incidence rate in Europe and America is 1:5000, and the incidence rate in China is 1:2300. [1-2] However, studies have shown that patients with pre-refractive surgery have a prevalence of up to 5\%. [3] And with the advancement of diagnostic methods, the incidence rate is on the rise. Because the current pathogenesis of keratoconus is not completely clear, it cannot be completely cured. Non-surgical treatment methods mainly include frame mirror and contact lens. The main surgical methods include corneal stroma implantation, corneal transplantation and corneal collagen cross-linking. In recent years, new treatments such as matrix regeneration, matrix lens transplantation, and scleral mirror have been intensively studied. This article aims to provide a brief overview of the advantages and disadvantages of various treatments in order to bring better treatment to patients with keratoconus.

\section{Pathogenesis}

The etiology of keratoconus has not yet been clarified, and Weed KH [4] studies that it a certain family genetic predisposition. Environmental factors and other external factors also have certain effects. For example, long-term wear of contact lenses can also cause hypoxia or even apoptosis of corneal cells, which eventually leads to changes in corneal structure. [5]

Davidson et al [6] summarized the pathogenesis of keratoconus into the following two theories: one is the corneal stroma theory: the progressive thinning of the patient's corneal 
stroma, including the reduction and arrangement of collagen fibers in the stroma, leading to a decrease in the biostability of the cornea; the second is the corneal epithelial theory:the proteolytic enzymes hydrolyze the Bowman layer, the corneal stroma layer and even the endothelial layer by external causes such as frequent contact with the corneal contact lens. Other studies have shown that the collagen in the corneal stroma of keratoconus is abnormally degraded, and its content is related to the increase of degrading enzymes in the matrix and the decrease of degrading enzyme inhibitors. and matrix metalloproteinase may play a crucial role in matrix degradation and thinning. [7] In addition, the risk of corneal clilation caused by refractive can also accelerate the progression of Keratoconus.

\section{Diagnosis}

The machines currently used in the diagnosis of keratoconus are mainly corneal topography, anterior segment analysis system, eye response analyzer, wavefront aberrometer and confocal microscope. [8] Clinical keratoconus is easy to diagnose. It can be diagnosed under the slit lamp according to typical symptoms such as Munson's sign, Fleischer's ring and Vogt's streak. However, for the subclinical keratoconus, the patient only shows a decrease in vision and can be corrected by wearing glasses, which is often easily missed.

\section{Treatment Method}

Because the pathogenesis of keratoconus has not been completely clear, at this stage, treatment can only be carried out from the process of strengthening the corneal tissue strength and delaying the thinning of the corneal stromal layer. Non-surgical and surgical therapies are generally used to reconstruct visual function.

\subsection{Non-surgical Therapy}

The most commonly method used clinically in patients with early keratoconus is the rigid gas permeable contact lens(RGP). After wearing the glasses, the gap between the corneal surface and the lens is completely filled with tears, reshaping a regular front refractive interface, maximally correcting the patient's irregular astigmatism and improving its visual quality. [9] However, whether RGP can control the progression of keratoconus is still no exact evidence. In addition, long-term friction between the top of the cornea cone and the lens increases the risk of corneal scarring. [10]

\subsection{Surgical Therapy}

\subsubsection{Intrastromal Corneal Ring Segments (ICRs)}

The corneal stroma ring is a semi-annular segment which made of polymethyl methacrylate. It has a certain stretching elasticity, which can shorten the arc length of the central region, thereby reducing the curvature of the central cornea and achieving the purpose of refractive correction. [11] Colin et al [12] reported for the first time that this method can be used to treat patients with keratoconus who cannot tolerate contact lenses. Traditional ICRs are artificially separated between the layers of the cornea, and the accuracy is poor. With the popularity of femtosecond lasers, the corneal stroma tunnel made by them makes the accuracy and safety of the surgery improved. [13] Abdullhamid [14] reported postoperative follow-up of ICRs in 56 patients with keratoconus in 87 eyes. The results showed that all patients had a preoperative UCVA of $1.38 \pm 0.37$, a postoperative 4 months of $0.58 \pm 0.32$, and a postoperative 16 months of $0.48 \pm 0.30$. It can be seen that the treatment of keratoconus by ICRs is a safe and effective method in the short term.

\subsubsection{Keratoplasty}

Penetrating keratoplasty (PKP) has been used in advanced keratoconus. With the development of technology, femtosecond laser gradually replaces the hand-made corneal bed, which overcomes the drawbacks of irregular astigmatism after traditional PKP. Zhou Hongjian et al [15] showed that femtosecond laser-assisted PKP was more effective than conventional PKP in reducing postoperative irregular astigmatism, and the difference was statistically significant $(\mathrm{P}<0.05)$. However, immunological rejection after PKP is easily to cause transplantation failure. [16-17]

Deep anterior lamellar keratoplasty (DALK) implants the donor corneal stroma full layer and epithelial tissue onto the posterior elastic layer of the recipient plant, which has less damage to the intraocular tissue and has reduced the possibility of transplant failure. It is more suitable for patients with turbid matrix but healthy endothelials. However, DALK has a high risk of perforation in the implanted beds during the operation, and the elastic layer is broken after the postoperative operation. [18] In recent years, femtosecond lasers have been used to produce corneal flaps by precisely cutting the corneal thickness, controlling the occurrence of intraoperative corneal perforation. Mosca et al [19] and Lu Yan et al [20] performed femtosecond laser-assisted DALK in 13 cases of keratoconus and 10 cases with keratoconus, no significant complications during operation or postoperative. Postoperative follow-up UCVA and BCVA were improved compared with preoperative, and corneal topography and corneal thickness examination were normal.

Kim et al [21] used a $60 \mathrm{kHz}$ IntraLase femtosecond laser to simulate different keratoplasty in pig eyes and observed the degree of endothelial damage at the margin of the graft. The results show that the degree of endothelial damage of femtosecond laser DALK is lower than that of femtosecond laser PKP.

\subsubsection{Corneal Collagen Cross-Linking (CXL)}

CXL is currently the only treatment that can effectively strengthen the corneal tension, delay or prevent the progression of keratoconus, and even reduce the curvature of the cornea to some extent. It was first confirmed by Spoerl et al that it was effective for keratoconus treatment. CXL irradiates the sensitizer riboflavin with ultraviolet light of 370 $\mathrm{nm}$ wavelength to excite riboflavin to a triplet state, generates 
a reactive oxygen species, induces chemical cross-linking reaction between amino groups of collagen fibers, thereby increasing the mechanical strength of collagen fibers and resistance to corneal expansion, maintaining corneal biomechanical stability. [22] Generally, the corneal thickness is greater than 400um, and below 400um may damage endothelial cells. At this time, the hypotonic riboflavin drip cornea can be used to make the swelling reach the standard, and then continue to perform CXL. Hafezi et al [23] treated 20 patients with keratoconus with this method, and no obvious complications occurred after operation.

Traditional CXL needs to remove epithelial tissue with a central corneal diameter of 5-9 mm under topical anesthesia. Postoperative infection, pain and haze may occur. Some scholars have suggested that CXL should be performed in the case of retaining the epithelium. In view of the problem of poor penetration of riboflavin in the presence of epithelial cells, riboflavin can be rapidly infiltrated into the corneal stroma by using iontophoresis or penetration enhancer or by injecting riboflavin into a matrix bag made of femtosecond laser.. However, studies have shown that the riboflavin penetration effect of the epithelium is not as good as the classic epithelialization. [24]

In recent years, many scholars have begun to experiment with a variety of surgical superposition therapy. Coskunseven et al [25] compared the efficacy of two groups of patients with CXL and ICRs, the effect of first ICRs and then CXL is better. Therefore, it is recommended to first use ICRs to shape the cornea, and then CXL to further enhance the mechanical strength of the collagen fibers. Patients who have undergone CXL can still correct their vision by wearing glasses or RGP.

\section{New Research Progress}

\subsection{Matrix Regeneration}

The corneal stroma is the main component of the corneal structure, accounting for $90 \%$ of the total corneal thickness. Therefore, the biomechanical and structural properties of the corneal stroma determine the physiology and function of the cornea. The matrix layer is rich in a variety of collagen fibers and is a major component of resistance to stress and deformation. The glycosaminoglycan has a low secretion level in the quiescent state. Once the cornea is damaged and is in a pathological state, collagen and medium can be synthesized and secreted in a large amount, and the wound repair of the corneal tissue is promoted, thereby maintaining the biomechanical stability of the cornea. [26] Patients with keratoconus have progressive thinning of the stromal layer, normal structure is destroyed, corneal physiological function is disordered, and the visual quality of patients is degraded. When the corneal microenvironment changes, such as corneal damage, stromal cells within the lesion range will have different degrees of apoptosis. Studies have shown that corneal stromal cells have stem cell characteristics and can be induced to differentiate into a variety of cells with other functions under specific conditions. [27] The differentiation into fibroblasts and myofibroblasts can effectively promote the repair of corneal structure and the recovery of normal function. In the transformation process, through the joint action of the two, the stability and pressure resistance of the corneal tissue damage after repaired are improved. Therefore, the use of stem cell characteristics of stromal cells to treat keratoconus and other corneal diseases has broad clinical application prospects, and it is worthy of further research.

\subsection{Matrix Lens Transplantation}

Corneal lens surgery was first proposed by Barraquer to improve the refractive state of the cornea. However, because the lens materials and surgical methods are relatively simple, patients have poor postoperative corrected visual, corneal opacity and other complications. In recent years, the focus of corneal lens surgery research is to find the best lens materials and surgical methods suitable for patients. During the operating of Small incision lenticule extraction (SMILE), the removed matrix lens is usually discarded. In recent years, the matrix lens has been found to be reusable, which brings a new research direction for the development of corneal lens surgery. The matrix lens can remodel the matrix function of patients with keratoconus, strengthen the biomechanical structure of the cornea, and improve the refractive state of the cornea. The stromal layer is rich in collagen, no blood vessel and lymphoid tissue, so the probability of immune rejection after transplantation is low, which overcomes the drawbacks of traditional keratoplasty. However, long-term efficacy and safety after surgery still need a lot of research to confirm. Pradhan et al [28] first used matrix lens transplantation for clinical use, transplanting a matrix lens taken from a SMILE high myopia patient (-10.50D) to another hyperopia (12.00D/-1.50D*155) Patients, the latter one-year follow-up UCVA and BCVA increased compared with preoperative, and no rejection occurred. Therefore, matrix lens transplantation will make a big difference in the treatment of keratoconus.

\subsection{Scleral Mirror}

The diameter of the rigid contact lens is $>12 \mathrm{~mm}$, which belongs to the generalized scleral lens, and the localization area is in the sclera. It originated at the end of the 19th century and was reported to have been used in the 1970s. Compared with ordinary RGP, its advantage is to avoid direct contact with the cornea, which can greatly alleviate the discomfort of wearing RGP, while avoiding pressure on the apex of the cornea, reducing the possibility of corneal scar formation, and tears can relieve dry eye symptoms in patients with filling the gap between the lens and the cornea. The scleral mirror has both the comfort of a soft contact lens and the visual quality of a rigid contact lens. It also corrects corneal high astigmatism and has the same effect on irregular astigmatism correction. In recent years, various studies have shown that scleral mirrors have great advantages for the treatment of ocular surface diseases. Barnett et al [29] reported the use of scleral mirror in 34 patients (48 eyes) with irregular astigmatism after PKP surgery, postoperative review of BCVA, $39.6 \%$ of the eyes 
reached $20 / 20$ and above, $91.7 \%$ of the eyes reached $20 / 40$ and above, and with no obvious complications, the results are similar to other scholars in this regard. Harthan [30] used a small scleral lens to treat Graves' eye disease. Follow-up found that small scleral mirror not only helps the patient's ocular surface recovery, but also improves the patient's visual quality. However, due to the large size of the scleral lens, it is not convenient for RGP to wear and remove, which may cause lens wear. In addition, the effect of scleral lens on the control of keratoconus progression has not yet become clear, which has become one of the reasons why many patients give up scleral mirror. Since the thickness of the scleral mirror is higher than that of the ordinary RGP lens, the oxygen exchange caused by tear exchange is less. How to improve the oxygen permeability of the lens is also one of the research priorities. For patients with keratoconus who are not well-dressed with RGP, scleral mirrors are also an alternative non-surgical treatment.

\section{Conclusion}

The keratoconus is a progressive ophthalmic disease. If the treatment is not timely, it can eventually lead to blindness and seriously affect the normal life of the patient. Because the cause of keratoconus has not been clarified, treatment is difficult and cannot be completely cured. It is generally accepted that wearing RGP is the best choice for non-surgical treatment when the patient's early astigmatism is low; In patients with moderate or severe desease, corneal collagen cross-linking can delay the progression of the lesion, and can continue to wear frame mirror or RGP for vision correction. When the cornea is scarred or turbid, a corneal transplant is required. In recent years, the application advantages of femtosecond laser in keratoconus treatment have gradually emerged, and it is expected to become a powerful tool for the treatment of keratoconus in the future. With the rapid development of modern medical technology, matrix regenerative, matrix lens transplantation and scleral mirror are also constantly observed and improved for the treatment of keratoconus. Once its effectiveness and long-term safety are confirmed, it will have a broad clinical application prospects.

\section{References}

[1] Wu Dongfang, Zhang Xiaolan, Qiu Lemei. Diagnosis and treatment of keratoconus. New progress in ophthalmology. 2012, 32(12):1197-1200

[2] Scheschy U, Scheschy H. Langzeit-Verlaufsbeobachtung des Keratoconus bei Unilateralität [J]. Spektrum Augenheilkd, 2014, 29(3): 109-117

[3] Xie Peiying. Prevalence and clinical symptoms of keratoconus [J]. China Optical Technology Magazine, 2010, 5:529

[4] Weed KH. The variable expression of keratoconus within monozygotic twins: Dundee university scottish keratoconus study(DUSKS). Contact Lens Anterior Eye 2006;29:123-126
[5] Tao X, Yu H, Zhang Y, et al. Role of corneal epithelium in riboflavin/ultraviolet-A mediated corneal cross-linking treatment in rabbit eyes [J]. Biomed Res Int. 2013, 2013:624563

[6] Davidson AE, Hayes S, Hardcastle AJ, et al. The pathogenesis of keratoconus. Eye. 2014, 28(2):189-195

[7] Zhang Y, Mao X, Schwend T, et al. Resistance of corneal RFUVA-cross-linked collagens and small leucine-rich proteoglycans to degradation by matrix metalloproteinases. Invest Ophthalmol Vis Sci 2013; 54( 2): 1014-1025

[8] Yang Yang, Jiang Aimin. Classification and early diagnosis of keratoconus. Chinese Journal of Ophthalmology. 2015, 5(4):213-218

[9] Qiao Shining. Therapeutic effect of rigid gas permeable contact lens on mild to moderate keratoconus. Shenzhen Journal of Integrated Traditional Chinese and Western Medicine. 2017, 27(2):6-8

[10] Wu Y, Tan Q, Zhang W, et al. Rigid gas-permeable contact lens related life quality in keratoconic patients with different grades of severity. Clin Exp Optom. 2015, 98(2): 150-154

[11] Linebarger EJ, Song D, Ruckhofer J, et al. Intaes: the intrastromal corneal ring [J]. Int Ophthalmol Clin. 2000, 40:199-208

[12] Colin J, Cochener B, Savary G, et al. INTACS inserts for treating keratoconus: one-year results [J]. ophthalmology. 2001, 108:1409-1414

[13] Tomalla M, Cagnolati W. Modern treatment options for the therapy of keratoconus [J]. Cont Lens Anterior Eye. 2007, 30( 1): 61-66

[14] Abdullhamid Alghamdi. Evaluation of clinical effectiveness of intrastromal corneal ring segments among keratoconus patients. Guoji Yanke Zazhi(Int Eye Sci)2018, 18(8): 1365-1369

[15] Zhou Hongjian, Wen Feng, Lu Bing, et al. Therapeutic effect of femtosecond laser assisted penetrating keratoplasty. International journal of Ophthalmology. 2014; 14( 10): $1822-1824$

[16] Liu H, Chen Y, Wang P, et al. Efficacy and safety of deep anterior lamellar keratoplasty, vs. penetrating keratoplasty for keratoconus:a Meta analysis. PLOS One. 2015, 10(1):1-14

[17] Jin AC, Min AL. Kim MS. Long-term outcomes of penetrating keratoplasty in keratoconus:analysis of the factors associated with final visual acuities. Int J Ophthalmol. 2014, 7(3):517-521

[18] Romano V, lovieno A, Parente G, et al. Long-term clinical out-comes of deep anterior lamellar keratoplasty in patients with keratoconus. Am J Ophthalmol. 2015. 159(3):505-511

[19] Mosca L, Fasciani R, Tamburelli C, et al. Femtosecond laser-assisted lamellar keratoplasty:Early results [J]. Cornea. 2008, 27:668-672

[20] Lu Yan, Duan Yixian, Chen Xianfei, et al. Femtosecond laser-assisted deep-layer bed study [J]. Chinese Journal of Ophthalmology. 2014, 50(4):267-272

[21] Kim JH, Choi SK, Lee D. The comparison of fomtosecond laser-assisted penetrating keratoplasty with conventional surgery in terms of endothelial safety: ex vivo study using porcine eyes [J]. Cornea. 2009, 28:812-816 
[22] Yildirim A, Cakir $\mathrm{H}$, Kara N, et al. CorneM collagen crosslinking for ectasia after laser in situ keratomilesis: long-term results. J Cataract Refract Surg, 2014, 40: $1591-1596$

[23] Hafezi F, Mrochen M, Iseli HP, Seiler T. Collagen cross linking with ultraviolet-A and hypoosmolar riboflavin solution in thin corneas [J]. J Cataract Refract Surg. 2009, 35( 4):621-624

[24] Krueger RR, Herekar S, Spoerl E. First proposed efficacy study of high versus standard irradiance and fractionated riboflavin/uhtraviolet a cross--linking with equivalent energy exposure. Eye Contact Lens. 2014, 40:353-357

[25] Coskunseven E, Jankov MR 2nd, Hafezi F, Atun S, Arslan E, Kymionis GD. Effect of treatment sequence in combined intrastromal corneal rings and corneal collagen crosslinking for keratoconus $[\mathrm{J}]$. J Cataract Refract Surg. 2009, 35( 12):2084-2091
[26] Li Tao, Lu Zhiyu, Yan Pisong, et al. Clinical application of corneal biomechanics. International Ophthalmology Overview. 2017, 41(6):409-413

[27] Lwigale PY, Cressy PA, Bronner -Fraser M. Corneal keratocytes retain neural crest progenitor cell properties $[\mathrm{J}]$. Dev Biol, 2005, 288:284-293

[28] Pradhan KR, Reinstein DZ, Carp GI. et al. Femtosecond laser-assisted keyhole endokeratophakia: correction of hyperopia by implantation of an allogeneie lenticule obtained by SMILE from a myopic donor [J]. J Refract Surg. 2013, 29(11): 777-782

[29] Melissa Barnett, Vivian Lien, Jennifer Y. Use of Scleral Lenses and Miniscleral Lenses After Penetrating Keratoplasty. Eye \& Contact Lens. 2016;42: 185-189

[30] Jennifer S. Harthan. Therapeutic use of mini-scleral lenses in a patient with Graves'ophthalmopathy. J Optom. 2014, 7(1):62-6 